\title{
Optical navigation of a DROP-IN gamma probe as a means to strengthen the connection between robot-assisted and radioguided surgery
}

Samaneh Azargoshasb ${ }^{1}$, Krijn H.M. Houwing ${ }^{1}$, Paul R. Roos ${ }^{1}$, Sven I. van Leeuwen ${ }^{1}$, Michael Boonekamp ${ }^{2}$, Elio Mazzone ${ }^{3,4}$, Kevin Bauwens ${ }^{4}$, Paolo Dell'Oglio ${ }^{1,4,5,6}$, F.W.B. van Leeuwen ${ }^{1,4,6}$, Matthias N. van Oosterom ${ }^{1,6}$

${ }^{1}$ Interventional Molecular Imaging-Laboratory, Department of Radiology, Leiden University Medical Center, Leiden, the Netherlands

${ }^{2}$ Innovation Center Medical Technology, Leiden University Medical Center, Leiden, the Netherlands

${ }^{3}$ Division of Oncology/Unit of Urology, URI, IRCCS Ospedale San Raffaele, Milan, Italy; Vita-Salute San Raffaele University, Milan, Italy

${ }^{4}$ ORSI Academy, Melle, Belgium

${ }^{5}$ Department of Urology, ASST Grande Ospedale Metropolitano Niguarda, Milan, Italy

${ }^{6}$ Department of Urology, Netherlands Cancer Institute-Antoni van Leeuwenhoek Hospital, Amsterdam, the Netherlands

First author: S. Azargoshasb, MSc. (PhD-candidate) (S.Azargoshasb@lumc.nl)*

Corresponding author: Dr. M.N. van Oosterom (m.n.van oosterom@lumc.nl) *

*Leiden University Medical Center, Radiology

Albinusdreef 2, 2300 RC Leiden

The Netherlands

+31-(0)715262042

Running title: Navigation of a DROP-IN gamma probe

Words: 2500

Funding: Financial support (NWO-TTW-VICl grant 16141) and hardware support (Eurorad S.A.). 


\section{ABSTRACT}

Rationale: With translation of the DROP-IN gamma probe, radioguidance has advanced into laparoscopic robot-assisted surgery. 'GPS-like' navigation further enhances the symbiosis between nuclear medicine and surgery. Therefore, we developed a fluorescence-video-based tracking method that integrates the DROP-IN with navigated-robotic surgery.

Methods: Fluorescent markers, integrated into the DROP-IN, were automatically detected using a daVinci Firefly laparoscope. Subsequently, a declipseSPECT-navigation platform calculated the DROP-IN's location within the surgical field. Using a phantom $(n=3)$, we pursued robotic-navigation on single-photon emission computed tomography/computed tomography (SPECT/CT), while intraoperative feasibility was validated during porcine surgery $(n=4)$.

Results: Video-based tracking allowed for navigation of the DROP-IN towards all lesions detected on SPECT/CT (external-iliac and common-iliac artery regions). Augmented-reality visualization in the surgical console, indicated the distance to these lesions in real-time, confirmed by the DROP-IN read-out. Porcine surgery underlined the feasibility of the concept.

Conclusions: Optical navigation of a DROP-IN probe provides a next step towards connecting nuclearmedicine with robotic surgery.

Keywords: Radioguided surgery; Robot-assisted surgery; Optical navigation; Image-guided surgery; Augmented reality 


\section{INTRODUCTION}

In the pursuit of precision surgery, robot-assisted approaches are gaining traction (e.g., prostatectomy and lymphatic dissections). An effective minimal-invasive approach does not only require well-engineered (robotic)-instruments, but also precise target definition. Interventional-molecular imaging can help achieve this: radioguided surgery is one of the most used image-guided surgery techniques (1). Unfortunately, the use of laparoscopic gamma probes is cumbersome with the robot (2). To overcome these limitations, tethered DROP-IN gamma and beta probes have been introduced for sentinel lymph node $(2,3)$ and prostate-specific membrane antigen (PSMA)-targeted resections $(4,5)$. These robottailored modalities allow the surgeon to autonomously position the detector to localize lesions during surgery. In areas with great anatomical complexity, road-maps provided by preoperative imaging (singlephoton emission computed tomography/computed tomography (SPECT/CT) or positron emission tomography/CT(PET/CT)) are considered a critical tool to provide insight in the number and location of surgical targets (6). Unfortunately, in the robotic DROP-IN procedures, real-time registration of such preoperative imaging information into the surgical view is challenging.

SPECT-based navigation of gamma probes could translate road-maps created at nuclear medicine to the surgical theatre (7). Unfortunately, traditional tool-tracking technologies cannot be used with a DROP-IN probe, where the tethered DROP-IN nature prohibits tracking with external optical-trackingsystems and metal parts of the robot setup degrade the accuracy of electromagnetic-tracking-systems $(8,9)$. Alternatively, video-based tracking concepts have been pursued $(8,9)$. Use of patterned-surface markers, which can be segmented from laparoscopic video-feed using traditional white-light imaging, has been explored for tethered ultrasound and gamma probes $(10,11)$. A concept that has already seen translation to the clinic (9). For such a tracking method, a direct line-of-sight has to be maintained between the DROP-IN and the laparoscope. However, Wild et al. have proposed that this can be improved with fluorescence-surface markers, where a direct line-of-sight is less easily impaired by smoke, water, blood, or use in combination with fluorescence guidance (12). In combination with multi-color fluorescence-imaging (13), use of fluorescence markers would also aid the integration of robotic navigation with bi-modal tracers (14).

In this study, we exploit real-time fluorescence-based optical tracking for navigation of a DROPIN gamma probe during robotic procedures in phantoms and porcine models. To increase the translational potential of this concept we used image-guided surgery technologies already available in the clinic (DROPIN (2), multi-color fluorescence (13), and declipseSPECT).

\section{METHODS}

\section{Tracking and Navigation Setup}

The SPECT-navigated DROP-IN gamma probe setup (Figure 1) consisted of:

1) A DROP-IN gamma probe (Eurorad S.A.) (3).

2) A Da Vinci Si robot (Intuitive Inc.) with Firefly fluorescence laparoscope. Recording the raw fluorescence video-feed, allowed for simultaneous visualization of fluorescein in yellow, indocyanine green (ICG) in pink, and the anatomical background in blue/black\&white (13). To allow for accurate calculations within the tracking software, the Firefly intrinsic- and extrinsiccamera properties were calibrated (15). 
3) A declipseSPECT navigation system (SurgicEye $\mathrm{GmbH}$ ) with near-infrared (NIR) optical tracking (Northern Digital Inc.), running customized tracking software.

A three-ring fluorescent-marker pattern was incorporated into the DROP-IN housing, fabricated out of medical-grade ultraviolet-curable adhesives comprising fluorescein. The fluorescent emissions were automatically detected based on color and shape. Using the known geometrical arrangement, the pose of the DROP-IN tip could be estimated (5 degrees-of-freedom) with respect to the Firefly laparoscope (Figure 1b). With one reference target attached to the Firefly and one to the patient (Figure 1a), the declipseSPECT was used to determine the pose of the Firefly and the patient within the operating room.

\section{Phantom Evaluation}

To study the concept of SPECT/CT-based navigation, a laparoscopic torso phantom was used (15), which contained bones, artery structures, and a radioactive model of pelvic lymph nodes ( $2 \mathrm{MBq}{ }^{99 \mathrm{~m}} \mathrm{Tc}$ each). Radioactive nodes were placed at different locations (i.e. external-iliac artery right and left, and commoniliac artery left) and three separate SPECT/CT-scans were acquired with the navigation-reference target fixated at the phantom's hip, allowing for registration of the scans in the operating room.

\section{Porcine Evaluation}

To investigate the DROP-IN tracking setup in a real-life surgical setting, 4 pigs underwent robot-assisted laparoscopic surgery. Surgical targets were created by depositing ICG ( $50 \mu \mathrm{L}, 2.5 \mathrm{mg} / \mathrm{mL}$ solution in saline) in the abdominal wall (average of 3 per pig). The tracer deposits were manually set as navigation targets to which the DROP-IN probe was navigated. Animal experiments were performed under approval of the local ethical committee.

\section{RESULTS}

\section{Phantom Evaluation}

Figure 2 illustrates the concept of performing SPECT-based navigation of the DROP-IN in the robot-assisted laparoscopic setting (supplementary Video). A navigation-enriched view of the abdomen was visible in the robotic console, including an augmented reality overlay of the lesion segmented from SPECT/CT. Maintaining a direct line-of-sight between the Firefly and the DROP-IN allowed for real-time calculation of the distance between the targeted lesion and the DROP-IN tip in $\mathrm{mm}$. The marker geometry allowed for a great range of maneuverability: at a $10 \mathrm{~cm}$ distance in-line with the laparoscope, DROP-IN tracking was feasible for $0-360^{\circ}$ (roll), $0-360^{\circ}$ (pitch), and $15-165 \& 195-345^{\circ}$ (yaw). The location of the lesion targets (i.e. external-iliac artery left and right, and common-iliac artery left) meant that the high maneuverability of the DROP-IN was instrumental for intraoperative detection (2). The audible feedback provided by the DROP-IN gamma probe, confirmed effective navigation.

\section{Porcine Evaluation}

Robotic surgery in porcine models was used to evaluate translation into an actual surgical setting (Figure 3 and supplementary Video). In this real-surgical environment, the fluorescein-markers on the DROP-IN probe remained clearly detectable (in yellow), both in white-light and fluorescence-imaging mode. ICG containing lesions were only visible in fluorescence-imaging mode (in pink) and could be detected simultaneous to the DROP-IN markers (Figure 3C-E). Uniquely, the fluorescent emissions of fluorescein $\left(\lambda_{\mathrm{em} \max }=515 \mathrm{~nm}\right)$ and ICG $\left(\lambda_{\mathrm{em} \max }=820 \mathrm{~nm}\right)$ could be excited simultaneously and the emissions could be 
distinguished by exploring the fluorescence-multiplexing capabilities of the Firefly camera (13). This meant the surgeon was also able to visually confirm the localized lesions during navigation. Despite that the excitation power in the NIR region with this Firefly is roughly 100 times higher than in the blue region of the spectrum (13), there was no clear difference in intensity observed between the fluorescein markers and ICG lesions. Bleaching of the fluorescein ring-markers was not observed within 1-hour experiments. In addition, problems with blood contamination of the DROP-IN markers were not observed, indicating the potential of fluorescence-based markers as discussed previously (12).

\section{DISCUSSION}

We investigated the first steps towards optical navigation of the DROP-IN gamma probe, a concept that further integrates interventional-nuclear medicine during robotic surgery. Visualizing the position of the DROP-IN with respect to predefined lesions is likely to help surgeons reduce intraoperative uncertainties about the target location and the gamma probe read-out. The translational character of the findings is underscored by: 1) the proven utility of the DROP-IN concept in prostate-cancer surgery (sentinel node-

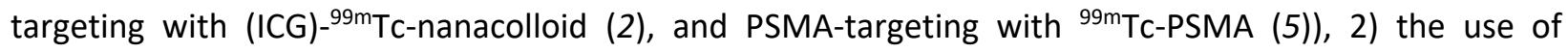
medical-grade fluorescent materials as markers, 3 ) the use of the clinically approved Firefly camera and declipseSPECT navigation system, and 4) a proof-of-concept in porcine surgery. Obviously, the concept could in the future be disseminated to alternative indications for which ${ }^{125} \mathrm{I},{ }^{99 \mathrm{~m}} \mathrm{Tc}$, or ${ }^{111} \mathrm{In}$-based radioguided surgery approaches have been reported (1), or even alternative multi-color fluorescence approaches (16).

In this study, we evaluated navigation in preoperative SPECT/CT-scans, but the same concept could also be expanded by using e.g. a DROP-IN beta (or even DROP-IN ultrasound) probe in combination with PET/CT-scans (4). Unfortunately, the surgical facilities that we used to test the presented technologies did not allow us to evaluate SPECT/CT-based navigation in porcine models. Since to date a facility allowing the latter experiment is unavailable, this link has to be further validated in clinical trials, as was done for other gamma probes (7). The current study, however, ensures that follow-up trials can rely on a validated technology, thus limiting the exposure of patients to unnecessary risks.

\section{CONCLUSION}

Surgical navigation of the DROP-IN probe based on SPECT/CT is promising, providing the next step towards precision-radioguided surgery, connecting nuclear-medicine with robotic surgery. In-human studies are in preparation to confirm these findings and evaluate oncological benefit of such an image-guided surgery approach.

\section{DISCLOSURE}

Funding by an NWO-TTW-VICl grant (\#16141), hardware support by Eurorad S.A.. MvO, FvL and KH have a pending-patent on fluorescent-marker tracking. During this research, EM, KB, PD and FvL were (partially) affiliated with ORSI Academy. No further conflicts of interest exist. 


\section{ACKNOWLEDGMENTS}

We acknowledge Danny van Willigen, Tessa Buckle (LUMC, the Netherlands) and the ORSI Academy staff (Belgium) for assistance during surgical evaluations, as well as the Skills-Lab and Petra Dibbets (LUMC) for assistance during phantom evaluations.

\section{KEY POINTS}

Question: Is it possible to navigate a DROP-IN gamma probe towards preoperatively marked lesions during robotic surgery?

Pertinent findings: As evaluated in a robot-assisted phantom setting, fluorescence markers can be used to navigate the DROP-IN towards lesions as identified on SPECT/CT. The feasibility of this concept was confirmed during surgery in porcine models.

Implications for patient care: Proving its utility in large animal models, a next step has been taken towards precision-radioguided surgery in the human robot-assisted setting. 


\section{REFERENCES}

1. Van Oosterom MN, Rietbergen DD, Welling MM, et al. Recent advances in nuclear and hybrid detection modalities for image-guided surgery. Expert Review of Medical Devices. 2019;16:711-734.

2. Dell'Oglio P, Meershoek P, Maurer T, et al. A DROP-IN Gamma Probe for Robot-assisted Radioguided Surgery of Lymph Nodes During Radical Prostatectomy. European Urology. 2021;79: 124132.

3. van Oosterom MN, Simon $\mathrm{H}$, Mengus L, et al. Revolutionizing (robot-assisted) laparoscopic gamma tracing using a drop-in gamma probe technology. American journal of nuclear medicine and molecular imaging. 2016;6:1.

4. Collamati F, van Oosterom MN, De Simoni M, et al. A DROP-IN beta probe for robot-assisted 68 Ga-PSMA radioguided surgery: first ex vivo technology evaluation using prostate cancer specimens. EJNMMI research. 2020;10:1-10.

5. van Leeuwen FW, van Oosterom MN, Meershoek P, et al. Minimal-invasive robot-assisted imageguided resection of prostate-specific membrane antigen-positive lymph nodes in recurrent prostate cancer. Clin Nucl Med. 2019;44:580-581.

6. Meershoek $\mathrm{P}$, Buckle $\mathrm{T}$, van Oosterom MN, et al. Can fluorescence-guided surgery help identify all lesions in unknown locations or is the integrated use of a roadmap created by preoperative imaging mandatory? A blinded study in prostate cancer patients. Journal of Nuclear Medicine. 2019:jnumed. 119.235234.

7. Bowles H, Sánchez N, Tapias A, et al. Radioguided surgery and the GOSTT concept: From preoperative image and intraoperative navigation to image-assisted excision. Revista Española de Medicina Nuclear e Imagen Molecular (English Edition). 2017;36:175-184.

8. Bernhardt S, Nicolau SA, Soler L, et al. The status of augmented reality in laparoscopic surgery as of 2016. Medical image analysis. 2017;37:66-90.

9. van Oosterom MN, van der Poel HG, Navab N, et al. Computer-assisted surgery: virtual-and augmented-reality displays for navigation during urological interventions. Current opinion in urology. 2018;28:205-213.

10. Fuerst B, Sprung J, Pinto F, et al. First robotic SPECT for minimally invasive sentinel lymph node mapping. IEEE Transactions on Medical Imaging. 2015;35:830-838.

11. Huang B, Tsai Y-Y, Cartucho J, et al. Tracking and visualization of the sensing area for a tethered laparoscopic gamma probe. International Journal of Computer Assisted Radiology and Surgery. 2020;15:1389-1397.

12. Wild E, Teber D, Schmid D, et al. Robust augmented reality guidance with fluorescent markers in laparoscopic surgery. International journal of computer assisted radiology and surgery. 2016;11:899-907.

13. Meershoek $P$, KleinJan $G H$, van Willigen DM, et al. Multi-wavelength fluorescence imaging with a da Vinci Firefly-a technical look behind the scenes. Journal of Robotic Surgery. 2020; epub.

14. van Leeuwen FW, Schottelius M, Brouwer OR, et al. Trending: Radioactive and fluorescent bimodal/hybrid tracers as multiplexing solutions for surgical guidance. Journal of Nuclear Medicine. 2020;61:13-19.

15. van Oosterom MN, Engelen MA, van den Berg NS, et al. Navigation of a robot-integrated fluorescence laparoscope in preoperative SPECT/CT and intraoperative freehand SPECT imaging data: a phantom study. Journal of biomedical optics. 2016;21:086008.

16. van Beurden $F$, van Willigen DM, Vojnovic $B$, et al. Multi-wavelength Fluorescence in image-guided surgery, clinical feasibility and future perspectives. Molecular Imaging. 2020; 19:1536012120962333. 


\section{FIGURES}

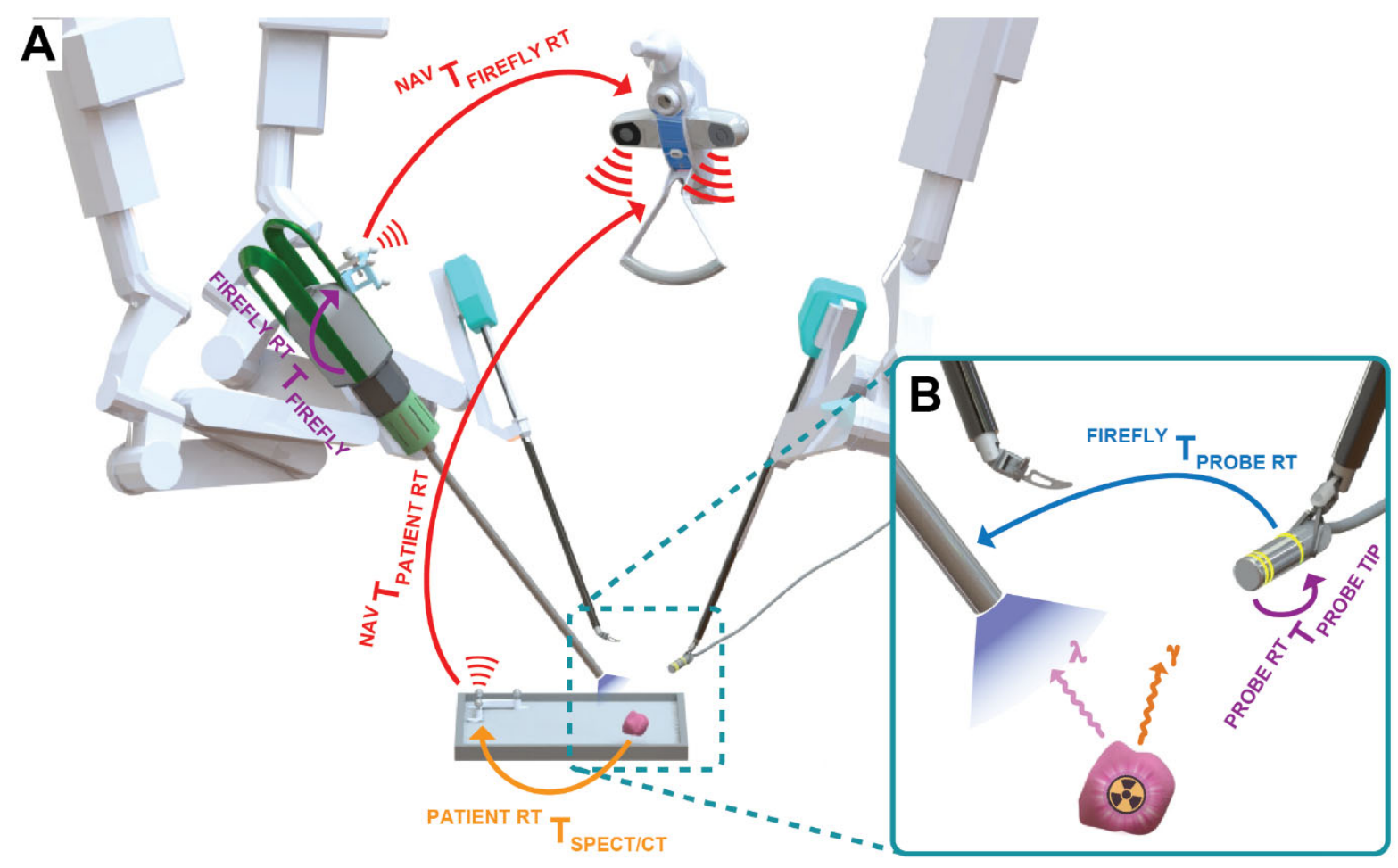

Figure 1. Overview of the DROP-IN tracking-and-navigation setup displaying the underlying poserelations with colored arrows. (A) Near-infrared optical tracking determines the pose of the Firefly and patient reference targets (RT; red arrows), while underlying registrations translate this to the Firefly camera (purple arrow) and lesions as found on SPECT/CT (orange arrow). (B) Vision-based tracking from the Firefly determines the pose of the DROP-IN markings within the surgical field (blue arrow), which are calibrated with the DROP-IN probe tip (purple arrow). Finally, this enables navigation of the DROP-IN towards the SPECT/CT-marked lesions. 

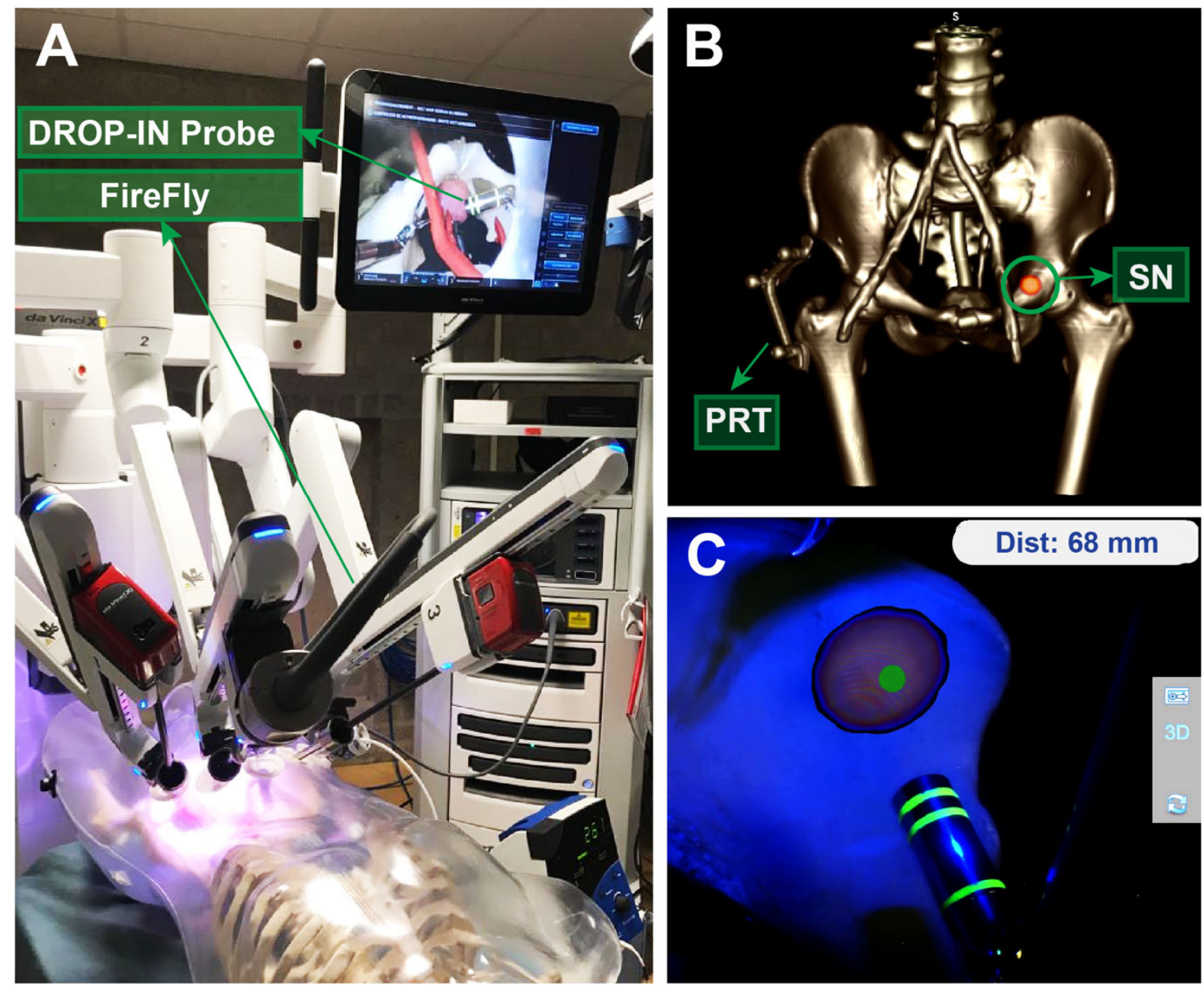

Figure 2. Real-time DROP-IN tracking and navigation during robot-assisted laparoscopic surgery in a phantom. (A) Setup overview. (B) Phantom SPECT/CT example, displaying the patient reference target (PRT) and targeted lymph node (SN). (C) Augmented reality visualization in the surgical console, displaying DROP-IN navigation towards the lymph node (including the calculated distance). 

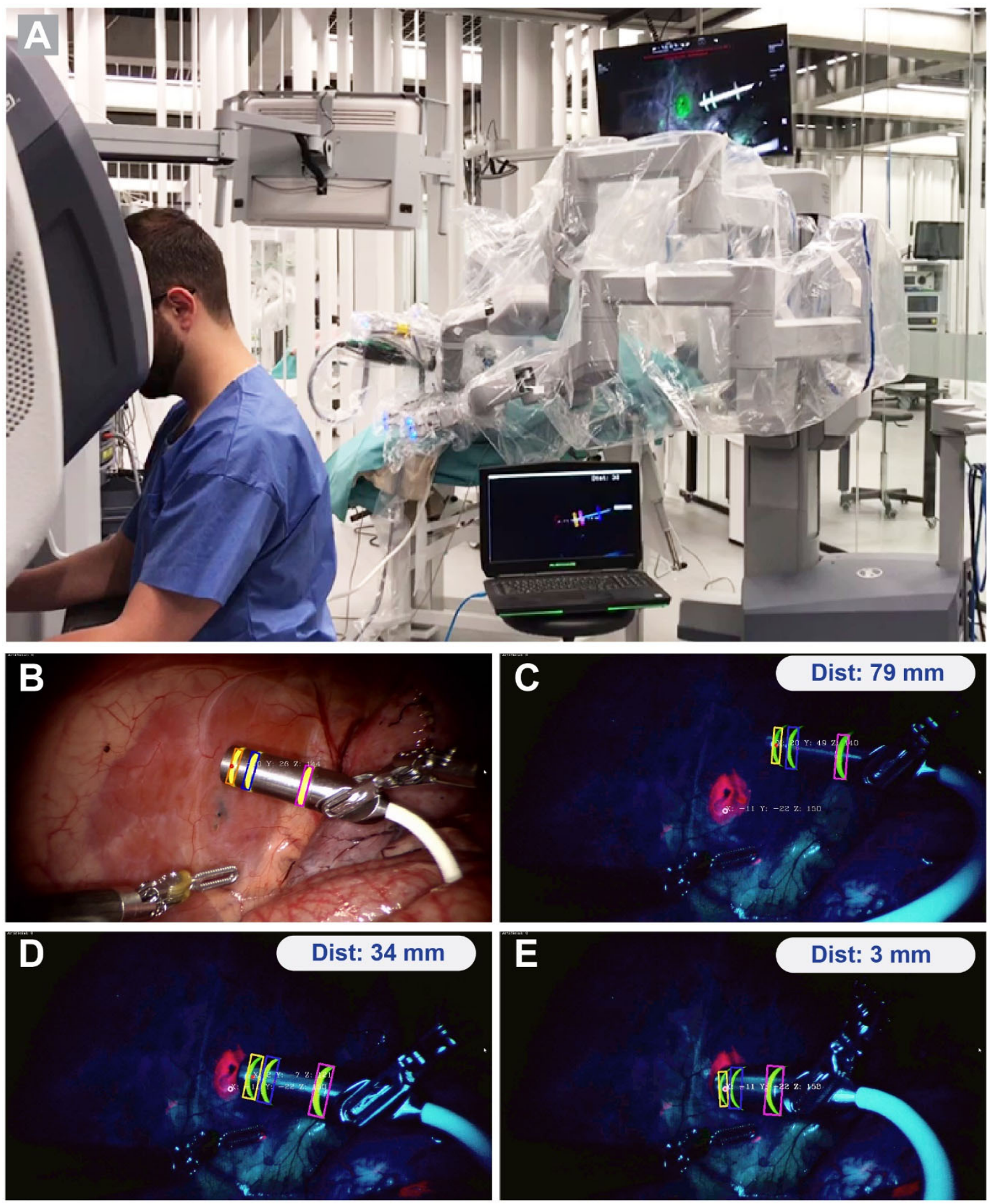

Figure 3. Real-time tracking of the DROP-IN probe with respect to targeted lesions during robotassisted laparoscopic surgery in pigs. (A) Operating room overview. (B) White-light imaging in the pig. (C-E) Tracking DROP-IN probe with respect to the targeted lesion (pink) using fluorescence-imaging settings, with the calculated target-distance visualized in the top-right-corner. 
GRAPHICAL ABSTRACT

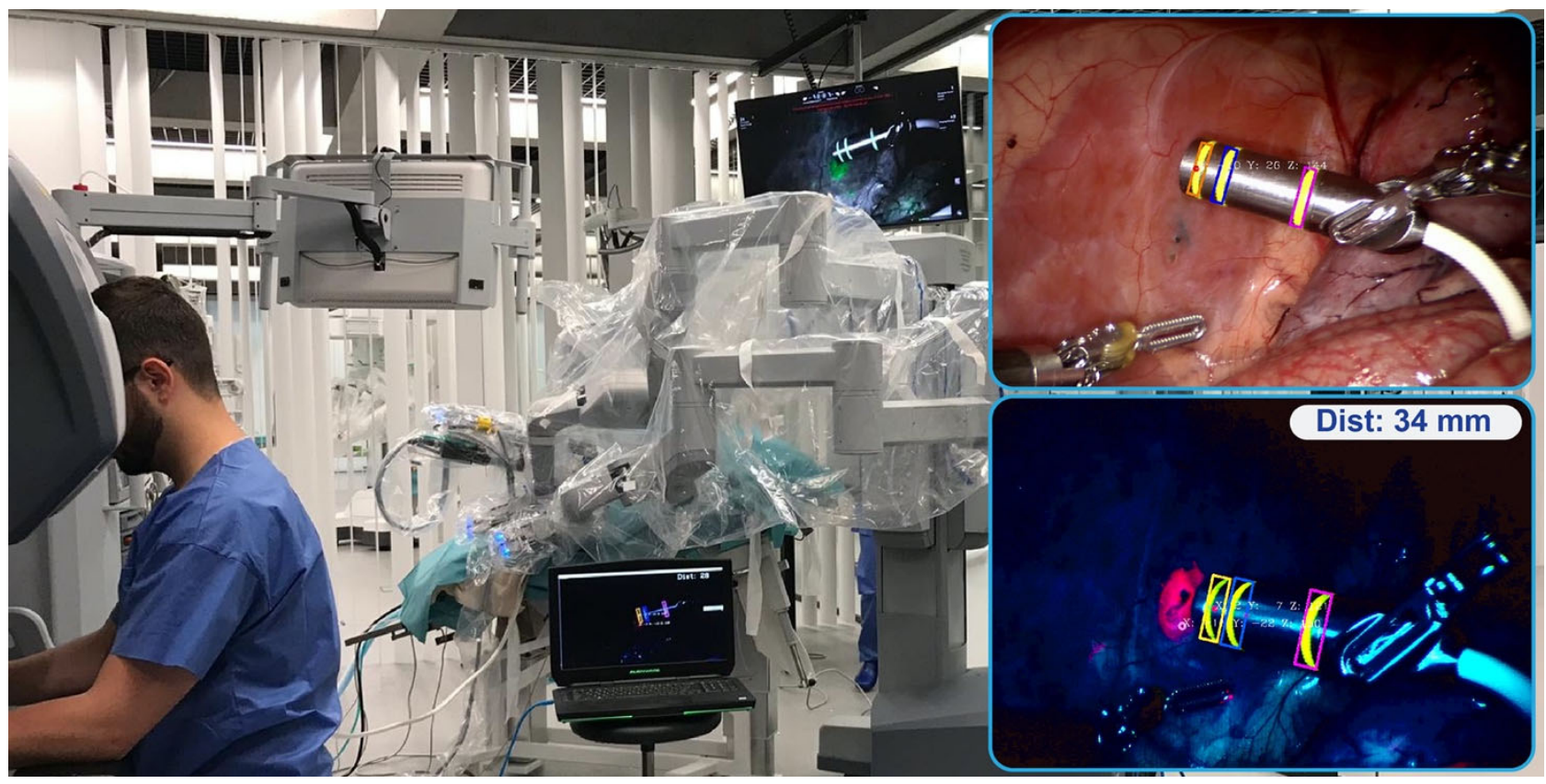

